\title{
Lower Lip and Chin Reconstruction with Functional Myocutaneous Gracilis Flap
}

\author{
Antonio Spaggiari' \\ Giorgio De Santis ${ }^{1}$ \\ 1Department of Plastic Surgery, Azienda Ospedaliero-Universitaria \\ di Modena, Modena, Italy
}

Address for correspondence Elisa Benanti, MD, Department of Plastic Surgery, Azienda Ospedaliero-Universitaria di Modena, via del Pozzo 71, 41124 Modena, Italy (e-mail: elisa.benanti@gmail.com).

\begin{abstract}
Keywords

- gracilis myocutaneous free flap

- human bite

- lip replantation

- lower lip reconstruction

Lower lip is an important anatomical unit in daily life activities, and its proper functional and aesthetic reconstruction is crucial. In the literature, both locoregional and microsurgical flaps have been described in lower lip reconstruction. Few authors have reported lower lip reconstruction with gracilis free flap. We describe a case of wide lower lip and chin avulsion caused by human bite reconstructed with an innervated gracilis free flap raised with its overlying skin paddle. The gracilis flap was harvested with a skin paddle of $7 \times 5 \mathrm{~cm}$, and vascular and nervous anastomoses were performed. At 9-month follow-up, an electromyography showed high muscle activities observed in the central part of the flap, and the patient achieved good oral functions reported with daily life activities. Raising the flap with the overlying skin paddle allowed us to avoid morbidity in other donor sites, avoid scar retraction of the skin graft on the gracilis that could limit its movement, and plan aesthetical refinements such as hair transplantation or tattoo of the beard on the skin.
\end{abstract}

\section{Introduction}

Total or subtotal lower lip defect has to be reconstructed because of the essential function of this unit in daily life activities. Restoring the proper function of the lower lip along with a good aesthetic result is an important challenge for surgeons. Local flaps are a valid option for the reconstruction of lower lip defects, but they are mainly used in patients with incomplete defects or when the general conditions of the patients do not allow them to undergo microsurgical procedures. ${ }^{1}$ Free flaps such as radial forearm palmaris-longus free flap ${ }^{2}$ or composite anterolateral thigh-fascia lata flap ${ }^{3}$ do not restore the motor function. Since 2000 , good functional results were reported by Ninkovic ${ }^{4}$ and others using an innervated free gracilis muscle flap for total or subtotal lower lip reconstruction.

We describe a single-stage functional reconstruction of a subtotal lower lip and mental defect with free myocutaneous gracilis flap. Raising the muscular flap with its overlying skin paddle gave us two important advantages of (1) avoiding possible scar retraction due to skin graft contraction

published online July 12, 2019
DOI https://doi.org/

10.1055/s-0039-1693511 ISSN 0970-0358. and (2) allowing further aesthetical refinements such as hair transplantation and/or better ink absorption for beard tattoo.

\section{Case History}

\section{Patient}

A 50-year-old man presented to the emergency room with a wide lower lip injury caused by a human bite that occurred 2 hours earlier, with avulsion of approximately $80 \%$ of the lip and $30 \%$ of the chin (-Fig. 1). The patient carried the avulsed tissue in an ice bag. Neither history of smoking nor comorbidity was reported.

\section{Surgical Procedure}

An emergency surgery was planned to perform a debridement of nonvital tissues and quantify the damage. We attempted a replantation of the avulsed tissue with microsurgical anastomosis of inferior labial vessels ( - Fig. 2). However, after 3 to 4 hours of good revascularization, an arterial microthrombosis occurred and the replanted tissue was removed. After 3 days, we planned a reconstruction with

\section{License terms}

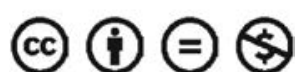




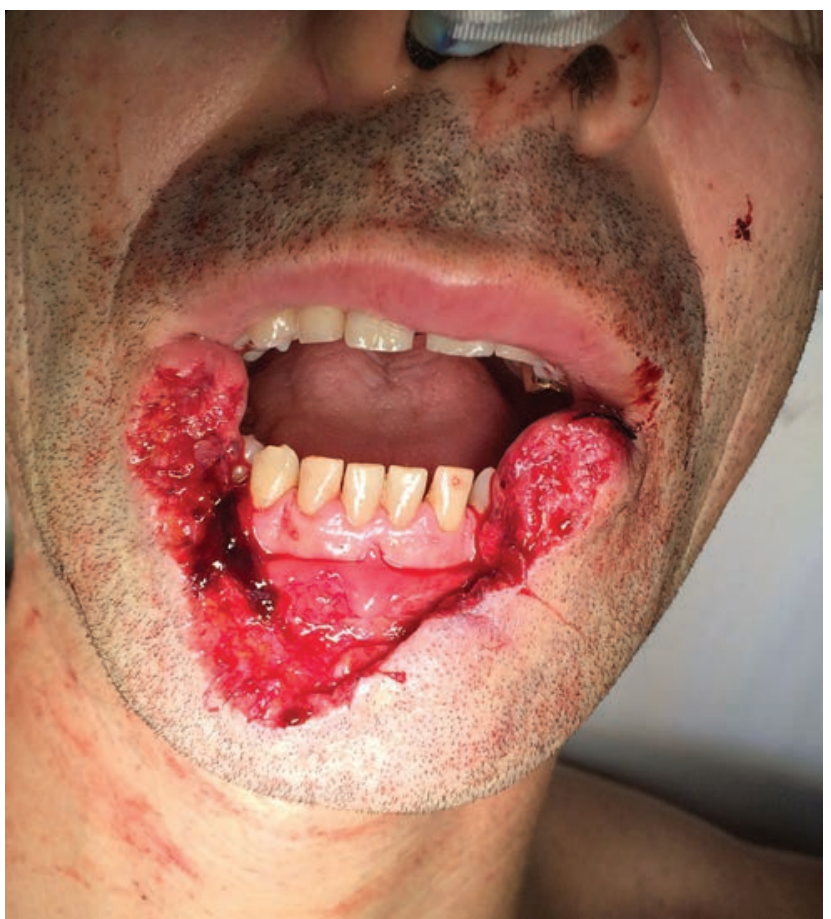

Fig. 1 Preoperative view of the defect.

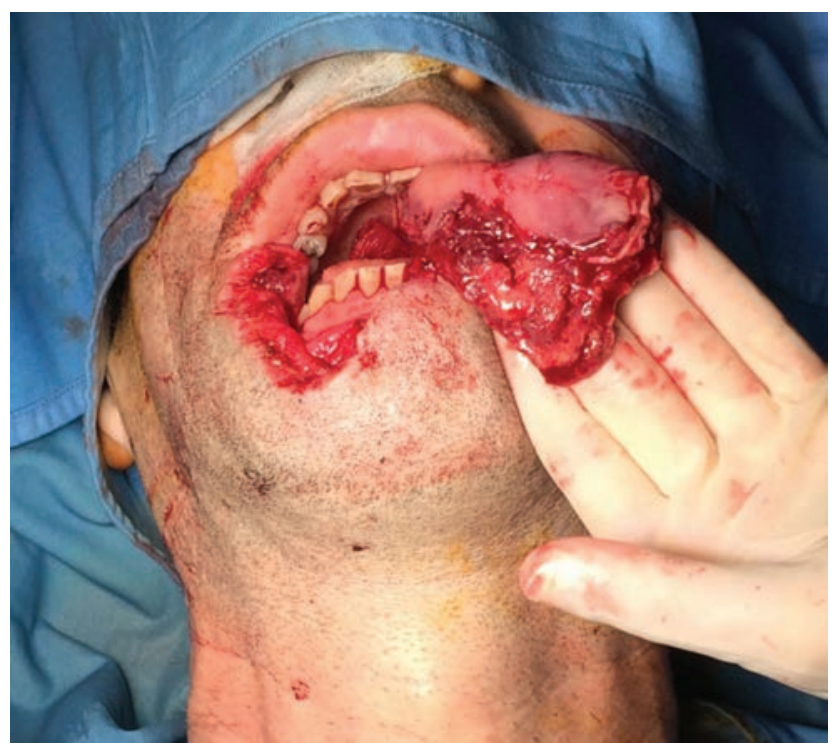

Fig. 2 Replantation of the avulsed lip.

myocutaneous gracilis flap. The gracilis flap was harvested with an oval-shaped skin paddle of $7 \times 5 \mathrm{~cm}$ centralized at the longitudinally oriented vessel pedicle (proximal onethird) compared with the muscle fibers ( - Figs. 3-4). Anastomosis of the vascular pedicle was performed with the facial vessels. While tailoring the flap, we have been careful to give the right tension to the muscle fibers ( - Fig. 5). Neural anastomosis was also performed between the obturator nerve and the nerve stump of the marginal mandibular nerve. The muscular layer of the flap was shaped for reconstruction of the inner part of the lip, whereas the skin paddle was outward placed. The patient was discharged 8 days after surgery.

\section{Results and Follow-Up}

At 1-month follow-up, the patient was able to open the mouth, laugh, and contain solid foods. After 6 months, the patient showed improvements in daily activities such as fluid containment, complete mouth closure, speaking, blowing, and whistling; complete mucosal metaplasia of the inner layer was observed. An electromyography was performed after 9 months: muscle activity was predominantly observed in the central part of the flap, with movements such as smiling, whistling, blowing, and lowering the lip. Concerning sensibility, the patient was able to identify deep pressure, and hot-and-cold discrimination test was positive. At 1-year follow-up, good thickness and quality of the skin paddle was observed, and hair transplantation could be now planned (-Fig. 6).

\section{Discussion}

Locoregional and microsurgical flaps were described in lower lip reconstruction. Among locoregional flaps, Karapandzic, bilateral Karapandzic, Webster, Bernard Burow, Gilles, and Millard flaps ${ }^{1}$ are local flaps that maintain sphincter function, but the complete recovery of sensitive and motor function is not always achieved and oral competence is not always assured. The deltopectoral flap ${ }^{1}$ and the submental flap were reported for total lower lip reconstruction, but the lack of sphincter function and the bulky aspect are the limitations in their use. ${ }^{5}$ Furthermore, they cannot be used for large defects. Free flaps can be used when the loss of lower lip involves more than two-thirds. Among the most selected free flaps, the radial forearm flap (alone or combined with palmaris longus) $)^{2}$ and the anterolateral thigh perforator flap ${ }^{3}$ do not allow a correct sphincter function reconstruction but only a mild oral competence. Dorsalis pedis flap, functional serratus anterior flap, and partial latissimus dorsi muscle flap did not gain much popularity. ${ }^{6,7}$ Since 2000, a few authors reported lower lip reconstruction with gracilis free flap harvested without the overlying skin paddle, and most of them used a combination with other free flaps for big facial injuries, but important donor-site morbidity was observed. ${ }^{8}$ Afterwards, Lengelé et al reported a prefabricated gracilis muscle free flap for a lower lip reconstruction, but a second surgical step was needed and scar contraction led to a loss of $25 \%$ of vestibular depth. ${ }^{9}$ The use of gracilis muscle to reconstruct lower lip defects has gained popularity since the study conducted by Ninkovic et al in $2007^{4}$ : they used the gracilis flap arranged with the facial artery musculomucosal flap for the mucosa restoration and used a skin graft harvested from the scalp for external skin. Afterward, Cordova et al added changes in the mucosal flap raising for the inner part of the lower lip reconstruction. ${ }^{10}$ The last report of this innervated free flap was in 2018 by Cakmak et al for a squamous cell carcinoma of the lower lip using a skin graft for the outside layer. ${ }^{11}$ As a first step, we attempted a replantation. Replantation of avulsed lower lip was successfully reported in 1988 by Schubert et al, ${ }^{12}$ but, in our case, probably the intimal damage of the 


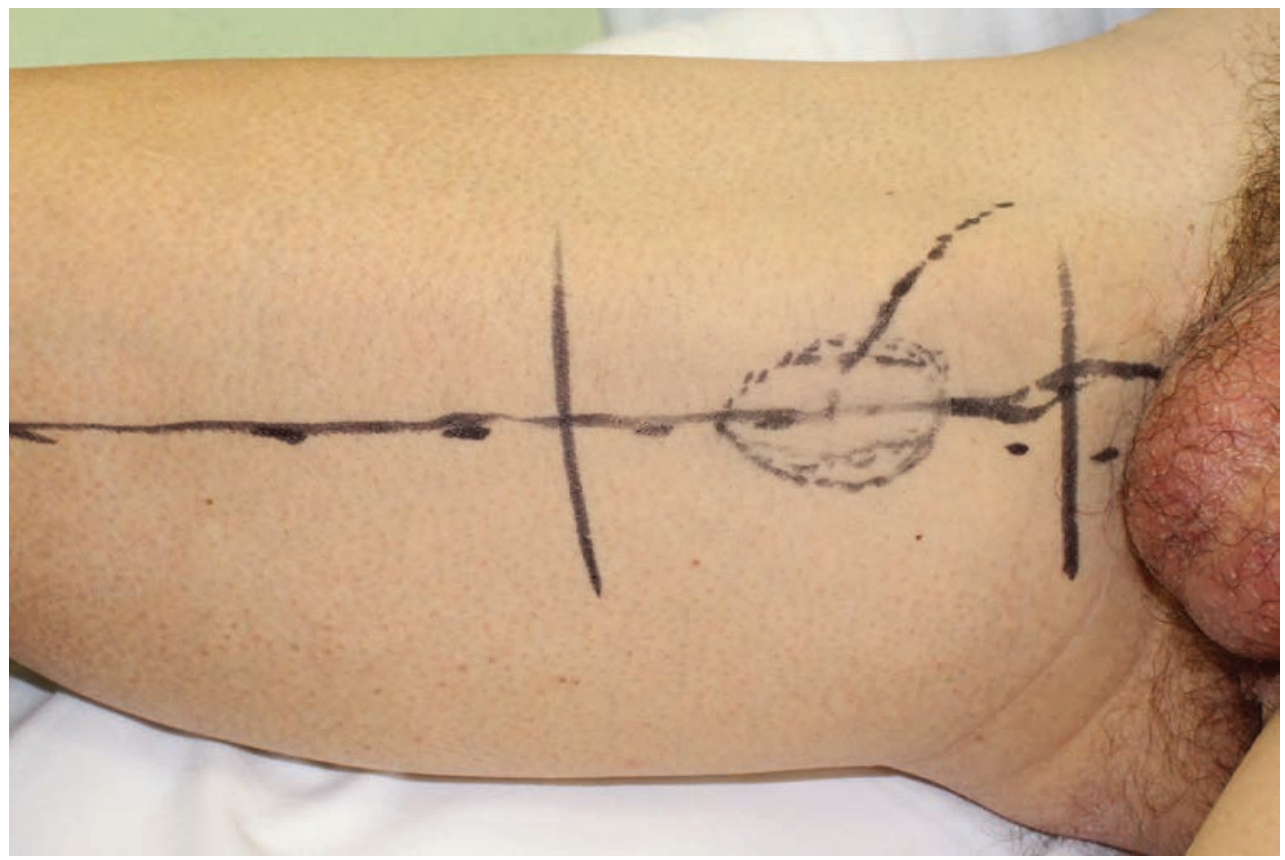

Fig. 3 Flap design.

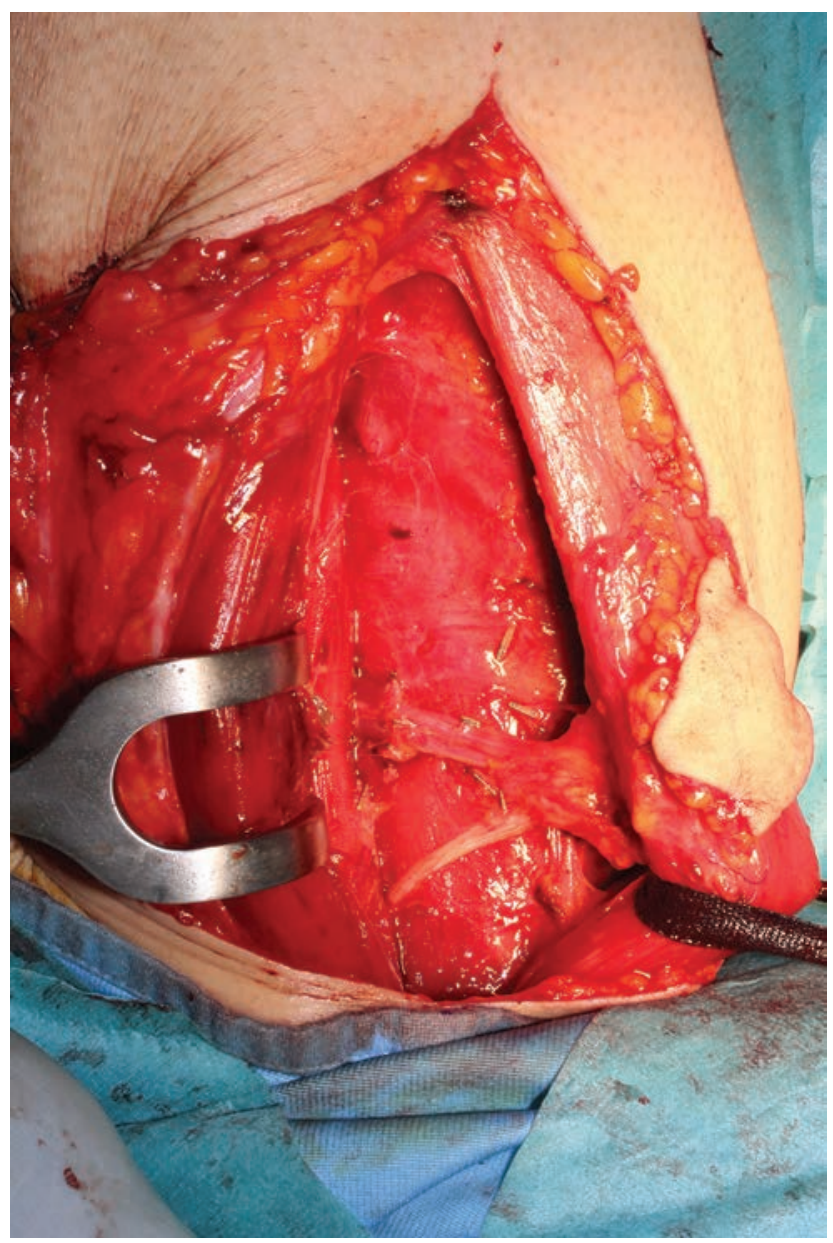

Fig. 4 Gracilis flap with the patient's overlying skin paddle.

artery caused by the tear of the bite led to microthrombi formation. We then chose an innervated myocutaneous gracilis flap to reconstruct the whole anatomical unit. The raising



Fig. 5 Shaping of the flap.

of the musculocutaneous flap avoided additional morbidity of another donor site such as skin grafts and possible scar retraction that could also limit the gracilis movements and function. For the inner mucosal layer, we did not use mucosal flaps, but we observed a complete mucosalization of the muscular flap. We achieved a good motor restoration, as electromyography and patient reported with daily life activities, and a good sensitive improvement even if no nerve anastomosis was performed probably due to sensitive nervous migration from the mucosa or from the mental nerve. In conclusion, we believe that by harvesting the skin paddle together with the muscle, it is possible to obtain better 


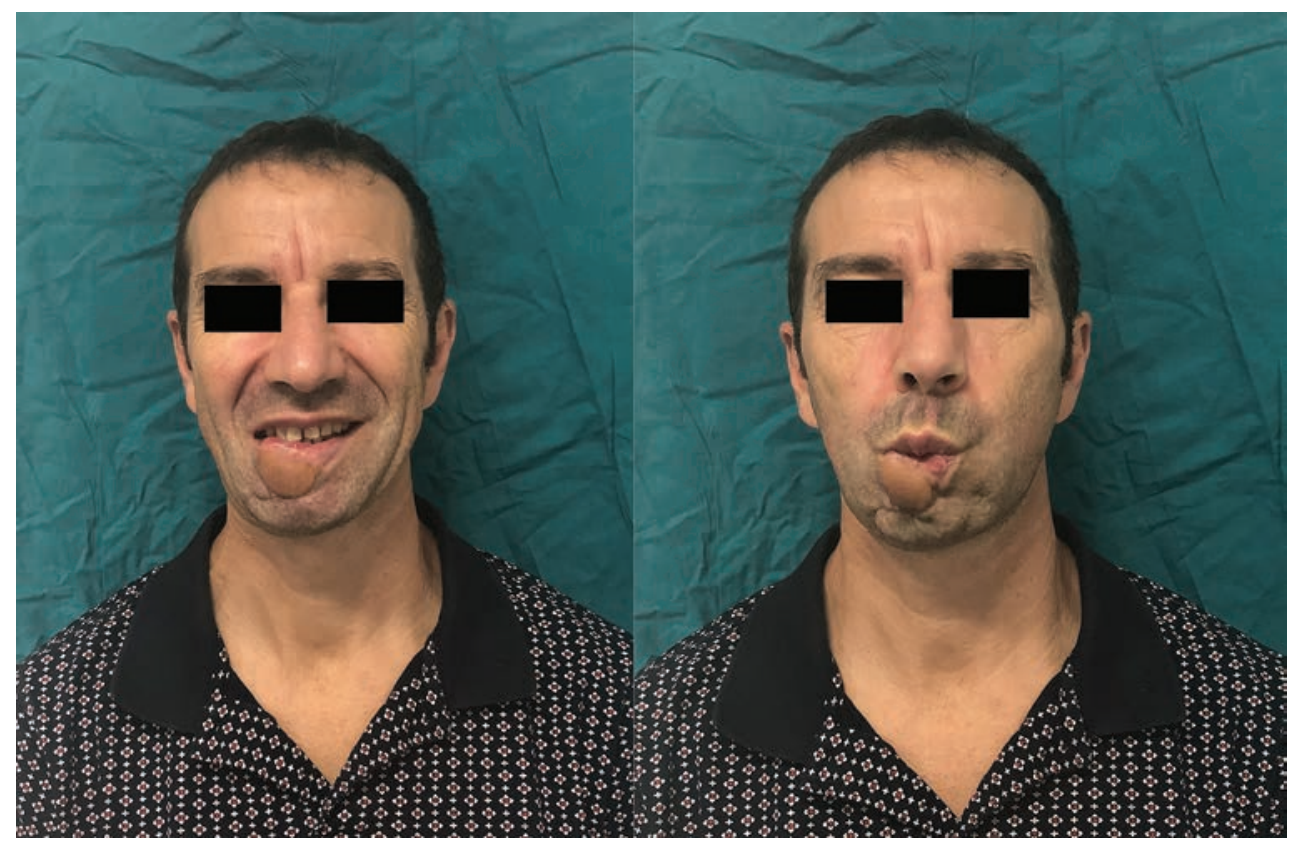

Fig. 6 One-year follow-up: patient smiling and blowing.

aesthetic and functional results since the muscle contracts more naturally and does not undergo retraction as in cases of skin grafting. Concerning aesthetic results, our approach allows us to avoid microstomia and big scars on the remaining healthy skin of the face as it occurs in lower lip reconstruction with locoregional flaps mostly in young patients with poor skin laxity. Furthermore, for the aesthetic purpose, hair transplantation or tattooing can be performed on the skin flap to restore the beard, and eventually a defatting of the flap can be easily achieved for better aesthetic results; however, the patient currently refuses to undergo these surgical refinements.

\section{Conflict of Interest}

None.

\section{References}

1 Baker SR. Regional flaps in facial reconstruction. Otolaryngol Clin North Am 1990;23(5):925-946

2 Sadove RC, Luce EA, McGrath PC. Reconstruction of the lower lip and chin with the composite radial forearm-palmaris longus free flap. Plast Reconstr Surg 1991;88(2):209-214

3 Yildirim S, Gideroğlu K, Aydogdu E, Avci G, Akan M, Aköz T. Composite anterolateral thigh-fascia lata flap: a good alternative to radial forearm-palmaris longus flap for total lower lip reconstruction. Plast Reconstr Surg 2006;117(6):2033-2041
4 Ninkovic M, Spanio di Spilimbergo S, Ninkovic M. Lower lip reconstruction: introduction of a new procedure using a functioning gracilis muscle free flap. Plast Reconstr Surg 2007;119(5):1472-1480

5 Koshima I, Inagawa K, Urushibara K, Moriguchi T. Combined submental flap with toe web for reconstruction of the lip with oral commissure. Br J Plast Surg 2000;53(7):616-619

6 Gundeslioglu AO, Karadag EC, Inan I, et al. Lip reconstruction using a functioning serratus anterior free flap: preliminary study. Int J Oral Maxillofac Surg 2017;46(10):1243-1247

7 Özkan Ö, Özkan Ö, Çinpolat A, et al. Functional lower lip reconstruction with the partial latissimus dorsi muscle free flap without nerve coaptation. Microsurgery 2019;39(2):131-137

8 Ueda K, Oba S, Nakai K, Okada M, Kurokawa N, Nuri T. Functional reconstruction of the upper and lower lips and commissure with a forearm flap combined with a free gracilis muscle transfer. J Plast Reconstr Aesthet Surg 2009;62(10):e337-e340

9 Lengelé BG, Testelin S, Bayet B, Devauchelle B. Total lower lip functional reconstruction with a prefabricated gracilis muscle free flap. Int J Oral Maxillofac Surg 2004;33(4):396-401

10 Cordova A, D’Arpa S, Moschella F. Gracilis free muscle transfer for morpho-functional reconstruction of the lower lip. Head Neck 2008;30(5):684-689

11 Cakmak MA, Cinal H, Barin EZ, Sakat MS, Karaduman H, Tan O. Total lower lip reconstruction with functional gracilis free muscle flap. J Craniofac Surg 2018;29(3):735-737

12 Schubert W, Kimberley B, Guzman-Stein G. Cunningham BL. Use of the labial artery for replantation of the lip and chin. Ann Plast Surg 1988;20(3):256-260 